\title{
Study of weak solutions for parabolic variational inequalities with nonstandard growth conditions
}

\section{Yan Dong*}

\section{"Correspondence:}

dongyan840214@126.com

Department of Basic, Shaanxi

Railway Institute, Weinan, China

\begin{abstract}
In this paper, we study the degenerate parabolic variational inequality problem in a bounded domain. First, the weak solutions of the variational inequality are defined. Second, the existence and uniqueness of the solutions in the weak sense are proved by using the penalty method and the reduction method.
\end{abstract}

MSC: 35B40; 35K35

Keywords: Parabolic variational inequality; Weak solution; Penalty method; Existence

\section{Introduction}

This article concerned with initial-boundary problem whose model is

$$
\begin{cases}\min \left\{L u, u(x, 0)-u_{0}(x)\right\}=0, & (x, t) \in Q_{T}, \\ u(x, t)=0, & (x, t) \in \Gamma_{T}, \\ u(x, 0)=u_{0}(x), & x \in \Omega,\end{cases}
$$

with

$$
L u=u_{t}-\operatorname{div}\left(a(u)|\nabla u|^{p(x, t)-2} \nabla u\right)-f(x, t), \quad a(u)=u^{\sigma}+d_{o},
$$

where $\Omega \subset \mathbb{R}^{+}$is a bounded simply connected domain, $Q_{T}=\Omega \times(0, T]$, and $\Gamma_{T}$ denotes the lateral boundary of the cylinder $Q_{T}$.

This type of variational inequality was studied initially by Chen and Yi [1], who proposed the equation

$$
\begin{cases}\frac{\partial}{\partial \tau} V-\frac{1}{2} \sigma^{2} \frac{\partial^{2}}{\partial x^{2}} V-\left(r-\frac{1}{2} \sigma^{2}\right) \frac{\partial}{\partial x} V+r V \geq 0 & \text { in } \Omega_{T}, \\ V \geq g(x), & \text { in } \Omega_{T}, \\ \left(\frac{\partial}{\partial \tau} V-\frac{1}{2} \sigma^{2} \frac{\partial^{2}}{\partial x^{2}} V-\left(r-\frac{1}{2} \sigma^{2}\right) \frac{\partial}{\partial x} V+r V\right)(V-g(x))=0 & \text { in } \Omega_{T}, \\ V(t, x)=0 & \text { on } \partial \Omega_{T}, \\ V(x, 0)=g(x) & \text { in } \Omega\end{cases}
$$

(c) The Author(s) 2018. This article is distributed under the terms of the Creative Commons Attribution 4.0 International License (http://creativecommons.org/licenses/by/4.0/), which permits unrestricted use, distribution, and reproduction in any medium, provided you give appropriate credit to the original author(s) and the source, provide a link to the Creative Commons license, and indicate if changes were made. 
for modeling the American option. When $r$ and $\sigma$ are positive constant, the existence and uniqueness of solutions to problem (4) were also studied in [2-4].

In 2014, the authors in [5] discussed the problem

$$
\begin{cases}u_{t}-L u-F(x, t, u, \nabla u) \geq 0 & \text { in } Q_{T}, \\ u(x, t) \geq u_{0}(x) & \text { in } Q_{T}, \\ \left(u_{t}-L u-F(x, t, u, \nabla u)\right)\left(u-u_{0}(x)\right)=0 & \text { in } Q_{T}, \\ u(x, 0)=u_{0}(x) & \text { on } \Omega, \\ u(x, t)=g(x) & \text { on } \partial \Omega \times(0, T)\end{cases}
$$

with second-order elliptic operator

$$
L(x, t)=\sum_{i, j=1}^{d} \frac{\partial}{\partial x_{j}}\left(a^{i j}(x, t) \frac{\partial}{\partial x_{i}}\right)-\sum_{i, j=1}^{d} b^{i}(x, t) \frac{\partial}{\partial x_{i}}-c(x, t) .
$$

They proved the existence and uniqueness of a solution to this problem with some conditions on $u_{0}, F$, and $L$. Later, the authors in $[6,7]$ extended the relative conclusions with the assumption that $a(u)$ and $p(x)$ are two positive constants. The author discussed the existence and uniqueness of a solution by the penalty method.

The existence and uniqueness of such a problem with the assumption that $p(x)$ and $a(u)$ are variables were less studied.

The aim of this paper is to study the existence and uniqueness of solutions for a degenerate parabolic variational inequality problem. Throughout the paper, we assume that the exponent $p(x, t)$ is continuous in $Q=\overline{Q_{T}}$ with logarithmic module of continuity:

$$
\begin{aligned}
& 1<p^{-}=\inf _{(x, t) \in Q} p(x, t) \leq p(x, t) \leq p^{+}=\sup _{(x, t) \in Q} p(x, t)<\infty, \\
& \forall z=(x, t) \in Q, \quad \xi=(y, s) \in Q_{T}, \quad|z-\xi|<1, \quad|p(z)-p(\xi)| \leq \omega(|z-\xi|),
\end{aligned}
$$

where

$$
\limsup _{\tau \rightarrow 0^{+}} \omega(\tau) \ln \frac{1}{\tau}=C<+\infty .
$$

The outline of this paper is as follows. In Section 2, we introduce the function spaces of Orlicz-Sobolev type, give the definition of a weak solution to the problem, and prove the existence and uniqueness. Section 3 is devoted to the proof of the existence and uniqueness of the solution obtained in Section 2.

\section{Basic spaces and the main results}

To study our problems, let us introduce the Banach spaces:

$$
\begin{aligned}
& L^{p(x, t)}\left(Q_{T}\right)=\left\{u(x, t) \mid u \text { is measurable in } Q_{T}, A_{p(\cdot)}(u)=\iint_{Q_{T}}|u|^{p(x, t)} \mathrm{d} x \mathrm{~d} t<\infty\right\}, \\
& \|u\|_{p(\cdot)}=\inf \left\{\lambda>0, A_{p(\cdot)}(u / \lambda) \leq 1\right\}, \\
& V_{t}(\Omega)=\left\{u\left|u \in L^{2}(\Omega) \cap W_{0}^{1,1}(\Omega),\right| \nabla u \mid \in L^{p(x, t)}(\Omega)\right\}, \quad\|u\|_{V_{t}(\Omega)}=\|u\|_{2, \Omega}+|\nabla u|_{p(\cdot) \Omega},
\end{aligned}
$$




$$
\begin{aligned}
& W\left(Q_{t}\right)=\left\{u:[0, T] \mapsto V_{t}(\Omega)\left|u \in L^{2}\left(Q_{t}\right),\right| \nabla u \mid \in L^{p(x, t)}\left(Q_{T}\right), u=0 \text { on } \Gamma_{T}\right\}, \\
& \|u\|_{W\left(Q_{t}\right)}=\|u\|_{2, Q_{T}}+|\nabla u|_{p(x, t), Q_{T}}
\end{aligned}
$$

and denote by $W^{\prime}\left(Q_{T}\right)$ the dual of $W\left(Q_{T}\right)$ with respect to the inner product in $L^{2}\left(Q_{T}\right)$.

In spirit of [3] and [4], we introduce the following maximal monotone graph:

$$
G(\lambda)= \begin{cases}0, & \lambda>0 \\ {[0,+\infty),} & \lambda=0\end{cases}
$$

In addition, we define the following function class for the solution:

$$
B=\left\{u \in W\left(Q_{T}\right) \cap L^{\infty}\left(0, T ; L^{\infty}(\Omega)\right)\right\} .
$$

Definition 2.1 A pair $(u, \xi) \in B \times L^{\infty}\left(\Omega_{T}\right)$ is called a weak solution of problem (1) if (a) $u(x, t) \leq u_{0}(x)$, (b) $u(x, 0)=u_{0}(x)$, (c) $\xi \in G\left(u-u_{0}\right)$, (d) for all $t_{1}, t_{2} \in[0, T]$, the following identity holds:

$$
\int_{t_{1}}^{t_{2}} \int_{\Omega}\left[u \varphi_{t}-\left(u^{\sigma}+d_{0}\right)|\nabla u|^{p(x, t)-2} \nabla u \nabla \varphi+f(x, t) \varphi-\xi \varphi\right] \mathrm{d} x \mathrm{~d} t=\left.\int_{\Omega} u \varphi \mathrm{d} x\right|_{t_{1}} ^{t_{2}} .
$$

The main theorem in this section is the following:

Theorem 2.1 Let $p(x, t)$ satisfy conditions (3)-(4). Suppose also that the following conditions hold:

$\left(H_{1}\right) \max \left\{1, \frac{2 N}{N+2}\right\}<p^{-}<N, 2 \leq \sigma<\frac{2 p^{+}}{p^{+}-1}$,

$\left(H_{2}\right) \quad u_{0} \geq 0, f \geq 0,\left\|u_{0}\right\|_{\infty, \Omega}+\int_{0}^{T}\|f(x, t)\|_{\infty, \Omega} \mathrm{d} t=K(T)<\infty$.

Then problem (1) has at least one weak solution in the sense of Definition 2.1.

Theorem 2.2 Suppose that the conditions in Theorem 2.1 are fulfilled and $p^{+} \geq 2$. Then problem (1) admits a unique solution in the sense of Definition 2.1 .

\section{Proof of the main results}

In this section, we consider the family of auxiliary parabolic problems

$$
\begin{cases}L_{\varepsilon} u_{\varepsilon}+\beta_{\varepsilon}\left(u_{\varepsilon}-u_{0}\right)=0, & (x, t) \in Q_{T}, \\ u(x, t)=\varepsilon, & (x, t) \in \Gamma_{T}, \\ u(x, 0)=u_{0}(x)+\varepsilon, & x \in \Omega .\end{cases}
$$

Here, $M$ is a positive parameter to be chosen later. Moreover,

$$
\begin{aligned}
& L_{\varepsilon} u_{\varepsilon}=\partial_{t} u_{\varepsilon}-\operatorname{div}\left(a_{\varepsilon, M}\left(u_{\varepsilon}\right)\left|\nabla u_{\varepsilon}\right|^{p(x, t)-2} \nabla u_{\varepsilon}\right)-f(x, t), \\
& 0<d_{0} \leq a_{\varepsilon, M}(u)=\left(\min \left(|u|^{2}, M^{2}\right)+\varepsilon^{2}\right)^{\frac{\sigma}{2}}+d_{0} \leq\left(M^{2}+1\right)+d_{0}, \quad 0<\varepsilon<1,
\end{aligned}
$$


and $\beta_{\varepsilon}(\cdot)$ is the penalty function satisfying

$$
\begin{array}{ll}
0<\varepsilon \leq 1, & \beta_{\varepsilon}(x) \in C^{2}(R), \quad \beta_{\varepsilon}(x) \leq 0, \quad \beta_{\varepsilon}(0)=-1, \\
\beta_{\varepsilon}^{\prime}(x) \geq 0, & \beta_{\varepsilon}^{\prime \prime}(x) \leq 0, \quad \lim _{\varepsilon \rightarrow 0} \beta_{\varepsilon}(x)= \begin{cases}0, & x>0, \\
-\infty, & x<0 .\end{cases}
\end{array}
$$

Following a similar method as in [6], we can prove that the regularized problem has a unique weak solution $u_{\varepsilon}(x, t) \in W\left(Q_{T}\right) \cap L^{2}\left(Q_{T}\right), \partial_{t} u_{\varepsilon}(x, t) \in W^{\prime}\left(Q_{T}\right)$ satisfying the following integral identities:

$$
\begin{gathered}
\int_{t_{1}}^{t_{2}} \int_{\Omega}\left[u_{\varepsilon} \varphi_{t}-a_{\varepsilon}, M\left(u_{\varepsilon}\right)\left|\nabla u_{\varepsilon}\right|^{p(x, t)-2} \nabla u_{\varepsilon} \nabla \varphi+f(x, t) \varphi\right] \mathrm{d} x \mathrm{~d} t \\
\quad=\int_{t_{1}}^{t_{2}} \int_{\Omega} \beta_{\varepsilon}\left(u_{\varepsilon}-u_{0}\right) \varphi \mathrm{d} x \mathrm{~d} t+\left.\int_{\Omega} u_{\varepsilon} \varphi \mathrm{d} x\right|_{t_{1}} ^{t_{2}}
\end{gathered}
$$

and

$$
\int_{t_{1}}^{t_{2}} \int_{\Omega}\left[\left(\partial_{t} u_{\varepsilon}\right) \varphi+a_{\varepsilon, M}\left(u_{\varepsilon}\right)|\nabla u|^{p(x, t)-2} \nabla u_{\varepsilon} \nabla \varphi-f(x, t) \varphi+\beta_{\varepsilon}\left(u_{\varepsilon}-u_{0}\right) \varphi\right] \mathrm{d} x \mathrm{~d} t=0 .
$$

We start with two preliminary results that will be used several times.

Lemma 3.1 Let $M(s)=|s|^{p(x, t)-2} s$. Then for all $\xi, \eta \in \mathrm{R}^{N}$,

$$
\begin{aligned}
& (M(\xi)-M(\eta))(\xi-\eta) \\
& \quad \geq \begin{cases}2^{-p(x, t)}|\xi-\eta|^{p(x, t)}, & 2 \leq p(x, t)<\infty, \\
(p(x, t)-1)|\xi-\eta|^{2}\left(|\xi|^{p(x, t)}+|\eta|^{p(x, t)}\right)^{\frac{p(x, t)-2}{p(x, t)}}, & 1<p(x, t)<2 .\end{cases}
\end{aligned}
$$

Lemma 3.2 (Comparison principle) Assume that $2<\sigma<\frac{2 p^{+}}{p^{+}-1}, p^{+} \geq 2$, and $u$ and $v$ are in $W\left(Q_{T}\right) \cap L^{\infty}\left(0, T ; L^{\infty}(\Omega)\right)$. If $L_{\varepsilon} u \geq L_{\varepsilon} v$ in $Q_{T}$ and if $u(x, t) \leq v(x, t)$ on $\partial Q_{T}$, then $u(x, t) \leq$ $v(x, t)$ in $Q_{T}$.

Proof We argue by contradiction. Suppose $u(x, t)$ and $v(x, t)$ satisfy $L_{\varepsilon} u \geq L_{\varepsilon} v$ in $Q_{T}$ and there is $\delta>0$ such that for $0<\tau \leq T, w=u-v>\delta$ on the set $\Omega_{\delta}=\Omega \cap\{x: w(x, t)>\delta\}$, and $\mu\left(\Omega_{\delta}\right)>0$. Let

$$
F_{\varepsilon}(\xi)= \begin{cases}\frac{1}{\alpha-1} \varepsilon^{1-\alpha}-\frac{1}{\alpha-1} \xi^{1-\alpha} & \text { if } \xi>\varepsilon \\ 0 & \text { if } \xi \leq \varepsilon\end{cases}
$$

where $\delta>2 \varepsilon>0$ and $\alpha=\frac{\sigma}{2}$. Let a test-function $\xi=F_{\varepsilon}(w) \in Z$ in (8). Then

$$
\begin{aligned}
0 \geq & \iint_{Q_{T}}\left[w_{t} F_{\varepsilon}(w)+\left(v^{\sigma}+d_{0}\right)\left(|\nabla u|^{p(x, t)-2} \nabla u-|\nabla v|^{p(x, t)-2} \nabla v\right) \nabla F_{\varepsilon}(w)\right] \mathrm{d} x \mathrm{~d} t \\
& +\iint_{Q_{T}}\left(u^{\sigma}-v^{\sigma}\right)|\nabla u|^{p(x, t)-2} \nabla u \nabla F_{\varepsilon}(w) \mathrm{d} x \mathrm{~d} t=J_{1}+J_{2}+J_{3},
\end{aligned}
$$


where $Q_{\varepsilon, \tau}=Q_{\tau} \cap\left\{(x, t) \in Q_{\tau} \mid w>\varepsilon\right\}$,

$$
\begin{aligned}
& J_{1}=\iint_{Q_{T}} w_{t} F_{\varepsilon}(w) \mathrm{d} x \mathrm{~d} t, \quad J_{2}=\iint_{Q_{T}}\left(u^{\sigma}-v^{\sigma}\right) w^{-\alpha}|\nabla u|^{p(x, t)-2} \nabla u \nabla w \mathrm{~d} x \mathrm{~d} t \\
& J_{3}=\iint_{Q_{T}}\left(v^{\sigma}+d_{0}\right) w^{-\alpha}\left(|\nabla u|^{p(x, t)-2} \nabla u-|\nabla v|^{p(x, t)-2} \nabla v\right) \nabla w \mathrm{~d} x \mathrm{~d} t .
\end{aligned}
$$

Now, let $t_{0}=\inf \{t \in(0, \tau]: w>\varepsilon\}$. Then we estimate $J_{1}, J_{2}$, and $J_{3}$ as follows:

$$
\begin{aligned}
J_{1} & =\iint_{Q_{\varepsilon, \tau}} w_{t} F_{\varepsilon}(w) \mathrm{d} x \mathrm{~d} t=\int_{\Omega}\left(\int_{0}^{t_{0}} w_{t} F_{\varepsilon}(w) \mathrm{d} t+\int_{t_{0}}^{\tau} w_{t} F_{\varepsilon}(w) \mathrm{d} t\right) \mathrm{d} x \\
& \geq \int_{\Omega} \int_{\varepsilon}^{w(x, \tau)} F_{\varepsilon}(s) \mathrm{d} s \mathrm{~d} x \geq \int_{\Omega_{\delta}} \int_{\varepsilon}^{w(x, \tau)} F_{\varepsilon}(s) \mathrm{d} s \mathrm{~d} x \\
& \geq \int_{\Omega_{\delta}}(w-2 \varepsilon) F_{\varepsilon}(2 \varepsilon) \mathrm{d} x \geq(\delta-2 \varepsilon) F_{\varepsilon}(2 \varepsilon) \mu\left(\Omega_{\delta}\right) .
\end{aligned}
$$

Let us first consider the case $p^{-} \geq 2$. By the first inequality of Lemma 3.1 we get

$$
\begin{aligned}
J_{2} & =\iint_{Q_{\varepsilon, \tau}}\left(v^{\sigma}+d_{0}\right) w^{-\alpha}\left(|\nabla u|^{p(x, t)-2} \nabla u-|\nabla v|^{p(x, t)-2} \nabla v\right) \nabla w \mathrm{~d} x \mathrm{~d} t \\
& \geq \iint_{Q_{\varepsilon, \tau}}\left(v^{\sigma}+d_{0}\right) w^{-\alpha} 2^{-p(x, t)}|\nabla w|^{p(x, t)} \mathrm{d} x \mathrm{~d} t \\
& \geq 2^{-p^{+}} \iint_{Q_{\varepsilon, \tau}}\left(v^{\sigma}+d_{0}\right) w^{-\alpha}|\nabla w|^{p(x, t)} \mathrm{d} x \mathrm{~d} t \geq 0 .
\end{aligned}
$$

Noting that $\frac{p(x, t)}{p(x, t)-1} \geq \frac{p^{+}}{p^{+}-1} \geq \frac{\sigma}{2}=\alpha>1$ and applying Young's inequality, we can estimate the integrand of $J_{3}$ in the following way:

$$
\begin{aligned}
& \left.\left|\left(u^{\sigma}-v^{\sigma}\right) w^{-\alpha}\right| \nabla w\right|^{p(x, t)-2} \nabla u \nabla w \mid \\
& \quad=\left.\left|\sigma w \int_{0}^{1}(\theta u+(1-\theta) v)^{\sigma-1} d \theta w^{-\alpha}\right| \nabla w\right|^{p(x, t)-2} \nabla u \nabla w \mid \\
& \left.\quad \leq \frac{C}{w^{\alpha}}\left[\frac{v^{\sigma}+d_{0}}{C}\right]|\nabla w|^{p(x, t)}+C_{1}\left(\sigma, d_{0}, K(T), p^{ \pm}\right)|w|^{p^{\prime}(x, t)}|\nabla u|^{p(x, t)}\right] \\
& \quad=\frac{\left(v^{\sigma}+d_{0}\right)}{2^{p^{+}+1} w^{\alpha}}|\nabla w|^{p(x, t)}+C_{1}\left(\sigma, d_{0}, K(T), p^{ \pm}\right)|w|^{p^{\prime}(x, t)-\alpha}|\nabla u|^{p(x, t)} \\
& \quad \leq \frac{\left(v^{\sigma}+d_{0}\right)}{2^{p^{+}+1} w^{\alpha}}|\nabla w|^{p(x, t)}+C_{1}\left(\sigma, d_{0}, K(T), p^{ \pm}\right)|\nabla u|^{p(x, t)}
\end{aligned}
$$

Substituting (12) into $J_{3}$, we get

$$
J_{3} \leq \frac{1}{2} J_{2}+C \iint_{Q_{\varepsilon, \tau}}|\nabla u|^{p(x, t)} \mathrm{d} x \mathrm{~d} t
$$


Second, we consider the case $1<p^{-} \leq p(x, t)<2, p^{+} \geq 2$. According to the second inequality of Lemma 3.1 , it is easily seen that the following inequalities hold:

$$
\begin{aligned}
J_{2} & =\iint_{Q_{\varepsilon, \tau}}\left(v^{\sigma}+d_{0}\right) w^{-\alpha}\left(|\nabla w|^{p(x, t)-2} \nabla u-|\nabla v|^{p(x, t)-2} \nabla v\right) \nabla w \mathrm{~d} x \mathrm{~d} t \\
& \geq\left(p^{-}-1\right) \iint_{Q_{\varepsilon, \tau}}\left(v^{\sigma}+d_{0}\right) w^{-\alpha}(|\nabla w|+|\nabla v|)^{p(x, t)-2}|\nabla w|^{2} \mathrm{~d} x \mathrm{~d} t .
\end{aligned}
$$

Using the conditions $1<\alpha \leq \frac{p^{+}}{p^{+}-1} \leq 2$ and Young's inequality, we can evaluate the integrand of $J_{3}$ as follows:

$$
\begin{aligned}
& \left.\left|\left(u^{\sigma}-v^{\sigma}\right) w^{-\alpha}\right| \nabla w\right|^{p(x, t)-2} \nabla u \nabla w \mid \\
& =\left.\left|\sigma w \int_{0}^{1}(\theta u+(1-\theta) v)^{\sigma-1} d \theta w^{-\alpha}\right| \nabla w\right|^{p(x, t)-2} \nabla u \nabla w \mid \\
& \leq \frac{\left(v^{\sigma}+d_{0}\right)\left(p^{-}-1\right)}{2 w^{\alpha}}(|\nabla w|+|\nabla v|)^{p(x, t)-2}|\nabla w|^{2} \\
& \left.\quad+C_{1}\left(\sigma, d_{0}, K(T), p^{ \pm}\right)|w|^{2-\alpha}|\nabla w|+|\nabla v|\right)^{p(x, t)} .
\end{aligned}
$$

Plugging (15) into $J_{3}$, we get

$$
\left.J_{3} \leq \frac{1}{2} J_{2}+C \iint_{Q_{\varepsilon, \tau}}|\nabla w|+|\nabla v|\right)^{p(x, t)} \mathrm{d} x \mathrm{~d} t
$$

Plugging estimates (10), (11), (13) and (10), (14), (16) into (9) and dropping the nonnegative terms, we arrive at the inequality

$$
(\delta-2 \varepsilon)\left(1-2^{1-\alpha}\right) \varepsilon^{1-\alpha} \mu\left(\Omega_{\delta}\right) \leq \tilde{C}
$$

with a constant $\tilde{C}$ independent of $\varepsilon$.

Notice that $\lim _{\varepsilon \rightarrow 0}(\delta-2 \varepsilon)\left(1-2^{1-\alpha}\right) \varepsilon^{1-\alpha} \mu\left(\Omega_{\delta}\right)=+\infty$, a contradiction. This means that $\mu\left(\Omega_{\delta}\right)=0$ and $w \leq 0$ a.e. in $Q_{\tau}$.

Lemma 3.3 Let $u_{\varepsilon}$ be weak solutions of (5). Then

$$
\begin{aligned}
& u_{0 \varepsilon} \leq u_{\varepsilon} \leq\left|u_{0}\right|_{\infty}+\varepsilon \\
& u_{\varepsilon_{1}} \leq u_{\varepsilon_{2}} \text { for } \varepsilon_{1} \leq \varepsilon_{2} .
\end{aligned}
$$

Proof First, we prove $u_{\varepsilon} \geq u_{0 \varepsilon}$ by contradiction. Assume that $u_{\varepsilon} \leq u_{0 \varepsilon}$ in $Q_{T}^{0}, Q_{T}^{0} \subset Q_{T}$. Noting that $u_{\varepsilon} \geq u_{0 \varepsilon}$ on $\partial Q_{T}$, we may assume that $u_{\varepsilon}=u_{0 \varepsilon}$ on $\partial Q_{T}^{0}$. With (5) and letting $t=0$, we deduce that

$$
\begin{aligned}
& L u_{0 \varepsilon}=\beta_{\varepsilon}\left(u_{0 \varepsilon}-u_{0 \varepsilon}\right)=-1, \\
& L u_{\varepsilon}=\beta_{\varepsilon}\left(u_{\varepsilon}-u_{0 \varepsilon}\right) \leq-1 .
\end{aligned}
$$


From Lemma 3.2 we conclude that

$$
u(x, t) \leq u_{0 \varepsilon}(x) \text { for any }(x, t) \in \Omega_{T},
$$

obtaining a contradiction.

Second, we pay attention to $u_{\varepsilon}(t, x) \leq\left|u_{0}\right|_{\infty}+\varepsilon$. Applying the definition of $\beta_{\varepsilon}(\cdot)$, we have

$$
L\left(\left|u_{0}\right|_{\infty}+\varepsilon\right)=0, \quad L u_{\varepsilon} \leq 0
$$

From (5) it is easy to prove that $u_{\varepsilon}(x, t) \geq \varepsilon$ on $\partial \Omega \times(0, T)$ and $u_{0 \varepsilon}(x) \geq \varepsilon$ in $\Omega$. Thus, combining (21) and (23) and repeating Lemma 3.3, we have

$$
u_{\varepsilon}(x, t) \geq \varepsilon \quad \text { in } \Omega \times(0, T) .
$$

Third, we aim to prove (19). Since

$$
\begin{aligned}
& L u_{\varepsilon_{1}}-\beta_{\varepsilon_{1}}\left(u_{0 \varepsilon_{1}}-u_{\varepsilon_{1}}\right)=0, \\
& L u_{\varepsilon_{2}}-\beta_{\varepsilon_{2}}\left(u_{0 \varepsilon_{2}}-u_{\varepsilon_{2}}\right)=0 .
\end{aligned}
$$

It follows by $\varepsilon_{1} \leq \varepsilon_{2}$ and the definition of $\beta_{\varepsilon}(\cdot)$ that

$$
\begin{aligned}
& \partial_{t} u_{\varepsilon_{2}}-L u_{\varepsilon_{2}}-\beta_{\varepsilon_{1}}\left(u_{0 \varepsilon_{1}}-u_{\varepsilon_{2}}\right) \\
& \quad=\beta_{\varepsilon_{2}}\left(u_{0 \varepsilon_{2}}-u_{\varepsilon_{2}}\right)-\beta_{\varepsilon_{1}}\left(u_{0 \varepsilon_{1}}-u_{\varepsilon_{2}}\right) \geq \beta_{\varepsilon_{2}}\left(u_{0 \varepsilon_{2}}-u_{\varepsilon_{2}}\right)-\beta_{\varepsilon_{1}}\left(u_{0 \varepsilon_{2}}-u_{\varepsilon_{2}}\right) \geq 0 .
\end{aligned}
$$

Thus, Lemma 3.3 can be proved by combining initial and boundary conditions in (5).

Moreover, with (18), we assert that there exists a subsequence $\varepsilon$ (still denoted by $\varepsilon$ ) such that

$$
\begin{aligned}
& u_{\varepsilon} \rightarrow u \in L^{p}\left(0, T ; W_{0}^{1, p}\left(\Omega_{T}\right)\right) \quad \text { as } \varepsilon \rightarrow 0, \\
& u_{\varepsilon} \geq u \geq 0 \quad \text { for any } \varepsilon>0 .
\end{aligned}
$$

Lemma 3.4 Let $u_{\varepsilon}$ be a solution of problem (5). For any $\varepsilon>0$, we have

$$
\left\|u_{\varepsilon}\right\|_{\infty, Q_{T}} \leq\left\|u_{0}\right\|_{\infty, \Omega}+\int_{0}^{T}\|f(x, t)\|_{\infty, \Omega} \mathrm{d} t=K(T)<\infty
$$

Proof Define

$$
u_{\varepsilon M}= \begin{cases}M & \text { if } u_{\varepsilon}>M, \\ u_{\varepsilon} & \text { if }\left|u_{\varepsilon}\right| \leq M, \\ -M & \text { if } u_{\varepsilon}<-M\end{cases}
$$


Choosing $u_{\varepsilon M}^{2 k-1}$ as a test-function in (8) and letting $t_{1}=t$ and $t_{2}=t+h$, we conclude that

$$
\begin{aligned}
& \frac{1}{2 k} \int_{t}^{t+h} \frac{\mathrm{d}}{\mathrm{d} t}\left(\int_{\Omega} u_{\varepsilon M}^{2 k} d x\right) \mathrm{d} t+\int_{t}^{t+h} \int_{\Omega}(2 k-1) a_{\varepsilon, M}\left(u_{\varepsilon M}\right) u_{\varepsilon M}^{2(k-1)}\left|\nabla u_{\varepsilon M}\right|^{p(x, t)} \mathrm{d} x \mathrm{~d} t \\
& \quad=\int_{t}^{t+h} \int_{\Omega} f u_{\varepsilon M}^{2 k-1} \mathrm{~d} x \mathrm{~d} t-\int_{t}^{t+h} \int_{\Omega} \beta_{\varepsilon} u_{\varepsilon M}^{2 k-1} \mathrm{~d} x .
\end{aligned}
$$

Letting $h \rightarrow 0$ and applying Lebesgue's dominated convergence theorem, we have that, for all $t \in(0, T)$,

$$
\begin{aligned}
& \frac{1}{2 k} \frac{\mathrm{d}}{\mathrm{d} t} \int_{\Omega} u_{\varepsilon M}^{2 k} \mathrm{~d} x+\int_{\Omega}(2 k-1) a_{\varepsilon, M}\left(u_{\varepsilon M}\right) u_{\varepsilon M}^{2(k-1)}\left|\nabla u_{\varepsilon M}\right|^{p(x, t)} \mathrm{d} x \\
& \quad=\int_{\Omega} f u_{\varepsilon M}^{2 k-1} \mathrm{~d} x-\int_{\Omega} \beta_{\varepsilon} u_{\varepsilon M}^{2 k-1} \mathrm{~d} x .
\end{aligned}
$$

Using Holder's inequality, we obtain

$$
\begin{aligned}
& \left|\int_{\Omega} f u_{\varepsilon M}^{2 k-1} d x\right| \leq\left\|u_{\varepsilon M}(\cdot, t)\right\|_{2 k, \Omega}^{2 k-1} \cdot\|f(\cdot, t)\|_{2 k, \Omega}, \quad k=1,2, \ldots \\
& \left|\int_{\Omega} \beta_{\varepsilon} u_{\varepsilon M}^{2 k-1} \mathrm{~d} x\right| \leq \int_{\Omega} u_{\varepsilon M}^{2 k-1} \mathrm{~d} x \leq\left\|u_{\varepsilon M}(\cdot, t)\right\|_{2 k, \Omega}^{2 k-1},
\end{aligned}
$$

whence

$$
\begin{gathered}
\left\|u_{\varepsilon M}\right\|_{2 k, \Omega}^{2 k-1} \frac{\mathrm{d}}{\mathrm{d} t}\left(\left\|u_{\varepsilon M}\right\|_{2 k, \Omega}\right)+(2 k-1) \int_{\Omega} a_{\varepsilon, M}\left(u_{\varepsilon M}\right) u_{\varepsilon M}^{2(k-1)}\left|\nabla u_{\varepsilon, M}\right|^{p(x, t)} \mathrm{d} x \\
\leq\left\|u_{\varepsilon M}\right\|_{2 k, \Omega}^{2 k-1} \cdot\|f(\cdot, t)\|_{2 k, \Omega}+C(T)\left\|u_{\varepsilon M}\right\|_{2 k, \Omega}^{2 k-1}, \quad k=1,2, \ldots
\end{gathered}
$$

By integration over $(0, t)$, for all $t$, we have

$$
\left\|u_{\varepsilon M}(\cdot, t)\right\|_{2 k, \Omega} \leq\left\|u_{\varepsilon M}(\cdot, 0)\right\|_{2 k, \Omega}+\int_{0}^{T}\|f\|_{2 k, \Omega} \mathrm{d} t+C(T), \quad \forall k \in \mathbb{N} .
$$

Then, as $k \rightarrow \infty$,

$$
\begin{aligned}
\left\|u_{\varepsilon M}(\cdot, t)\right\|_{\infty, \Omega} & \leq\left\|u_{\varepsilon M}(\cdot, 0)\right\|_{\infty, \Omega}+\int_{0}^{T}\|f\|_{\infty, \Omega} \mathrm{d} t \\
& \leq\left\|u_{0}\right\|_{\infty, \Omega}+\int_{0}^{T}\|f\|_{\infty, \Omega} \mathrm{d} t+C(T)=K(T)
\end{aligned}
$$

If we chose $M>K(T)$ then $u_{\varepsilon M}(\cdot, t) \leq \sup \left|u_{\varepsilon M}(\cdot, t)\right| \leq K(T)<M$, and therefore $u_{\varepsilon M}(\cdot, t)=$ $u_{\varepsilon}(\cdot, t)$.

Corollary 3.1 Choosing M large enough, we have

$$
\min \left\{u_{\varepsilon}^{2}, M^{2}\right\}=u_{\varepsilon}^{2} \quad \text { and } \quad a_{\varepsilon, M}\left(u_{\varepsilon M}\right)=a_{\varepsilon, M}\left(a_{\varepsilon}\right)=\left(\varepsilon^{2}+u_{\varepsilon}^{2}\right)^{\sigma / 2}+d_{0}
$$

Corollary 3.2 If $u_{0} \geq 0$ and $f \geq 0$, then the solution $u_{\varepsilon}(x, t)$ is nonnegative in $Q_{T}$. 
Proof Set $u_{\varepsilon}^{-}=\min \left\{u_{\varepsilon}, 0\right\}$. Then $u_{\varepsilon}^{-}(x, 0)=0, u_{\varepsilon}^{-} \mid \Gamma_{T}=0$, and

$$
\frac{1}{2} \frac{\mathrm{d}}{\mathrm{d} t}\left(\left\|u_{\varepsilon}^{-}(x, t)\right\|_{2, \Omega}^{2}\right)+\int_{\Omega} a_{\varepsilon, M}\left(u_{\varepsilon}\right)\left|\nabla u_{\varepsilon}^{-}\right|^{p(x, t)} \mathrm{d} x \leq 0 .
$$

It follows that, for every $t>0$,

$$
\left\|u_{\varepsilon}^{-}(x, t)\right\|_{2, \Omega} \leq\left\|u_{\varepsilon}^{-}(\cdot, 0)\right\|_{2, \Omega}=0
$$

and the required assertion follows.

Lemma 3.5 The solution of problem (5) satisfies the estimates

$$
\begin{aligned}
& \iint_{Q_{T}} u_{\varepsilon}^{\sigma}\left|\nabla u_{\varepsilon}\right|^{p(x, t)} \mathrm{d} x \mathrm{~d} t \leq K(T)|\Omega|^{\frac{1}{2}}, \\
& \varepsilon^{\sigma} \iint_{Q_{T}}\left|\nabla u_{\varepsilon}\right|^{p(x, t)} \mathrm{d} x \mathrm{~d} t \leq K(T)|\Omega|^{\frac{1}{2}}, \\
& d_{0} \iint_{Q_{T}}\left|\nabla u_{\varepsilon}\right|^{p(x, t)} \mathrm{d} x \mathrm{~d} t \leq K(T)|\Omega|^{\frac{1}{2}} .
\end{aligned}
$$

Proof Similarly as in Lemma 3.4, we take $k=1$ in (32) to get

$$
\frac{\mathrm{d}}{\mathrm{d} t}\left\|u_{\varepsilon}(\cdot, t)\right\|_{2, \Omega}+\int_{\Omega} a_{\varepsilon, M}\left(u_{\varepsilon}\right)\left|\nabla u_{\varepsilon}\right|^{p(x, t)} \mathrm{d} x \leq\|f\|_{2, \Omega}+C(T), \quad \forall t \in(0, T) .
$$

Clearly, integrating over $(0, t)$, we have

$$
\left\|u_{\varepsilon}(\cdot, t)\right\|_{2, \Omega}+\int_{0}^{t} \int_{\Omega} a_{\varepsilon, M}\left(u_{\varepsilon}\right)\left|\nabla u_{\varepsilon}\right|^{p(x, t)} \mathrm{d} x \mathrm{~d} t \leq\left\|u_{\varepsilon}(\cdot, t)\right\|_{2, \Omega}+\int_{0}^{T}\|f\|_{2, \Omega} \mathrm{d} t+C(T)
$$

Note that the first term on the left-hand side is nonnegative. We drop the nonpositive term in (37) to get

$$
\int_{0}^{t} \int_{\Omega} a_{\varepsilon, M}\left(u_{\varepsilon}\right)\left|\nabla u_{\varepsilon}\right|^{p(x, t)} \mathrm{d} x \mathrm{~d} t \leq K(T)|\Omega|^{\frac{1}{2}}
$$

If $a_{\varepsilon, M}\left(u_{\varepsilon}\right) \geq d_{0}$, then we have inequality (36), and if $a_{\varepsilon, M}\left(u_{\varepsilon}\right) \geq \varepsilon^{\sigma}$, then we have inequality (35) such that $M>K(T)$, and we have $a_{\varepsilon, M}\left(u_{\varepsilon}\right) \geq u_{\varepsilon}^{\sigma}$. Furthermore, we get inequality (34).

Lemma 3.6 The solution of problem (5) satisfies the estimate

$$
\left\|u_{\varepsilon t}\right\|_{W^{\prime}\left(Q_{T}\right)} \leq C\left(\sigma, p^{ \pm}, K(T),|\Omega|\right) .
$$

Proof From identity (7) we get

$$
\begin{aligned}
\iint_{Q_{T}} u_{\varepsilon t} \xi \mathrm{d} x \mathrm{~d} t= & -\iint_{Q_{T}}\left[\left(u_{\varepsilon}^{\sigma}+\varepsilon^{2}\right)^{\sigma / 2}+d_{0}\right]\left|\nabla u_{\varepsilon}\right|^{p(x, t)-2} \nabla u_{\varepsilon} \nabla \xi \mathrm{d} x \mathrm{~d} t \\
& +\iint_{Q_{T}} f(x, t) \xi(x, t) \mathrm{d} x \mathrm{~d} t-\iint_{Q_{T}} \beta_{\varepsilon}(x, t) \xi(x, t) \mathrm{d} x \mathrm{~d} t .
\end{aligned}
$$


Applying the fact that $\beta_{\varepsilon}(x, t) \in(0,1)$, we get

$$
\begin{aligned}
\iint_{Q_{T}} u_{\varepsilon t} \xi \mathrm{d} x \mathrm{~d} t \leq & \int_{0}^{T} \int_{\Omega}\left[\left(u_{\varepsilon}^{\sigma}+\varepsilon^{2}\right)^{\sigma / 2}+d_{0}\right]\left|\nabla u_{\varepsilon}\right|^{p(x, t)-1} \nabla u_{\varepsilon} \nabla \xi \mathrm{d} x \mathrm{~d} t \\
& +\int_{0}^{T} \int_{\Omega}|f+1| \cdot|\xi| \mathrm{d} x \mathrm{~d} t .
\end{aligned}
$$

Using the Hölder inequality repeatedly, we have that

$$
\begin{aligned}
\iint_{Q_{T}} u_{\varepsilon t} \xi \mathrm{d} x \mathrm{~d} t \leq & 2\left\|\left[\left(u_{\varepsilon}^{\sigma}+\varepsilon^{2}\right)^{\sigma / 2}+d_{0}\right]\left|\nabla u_{\varepsilon}\right|^{p(x, t)-1}\right\|_{p^{\prime}(x, t)}\|\nabla \xi\|_{p(x, t)} \\
& +2\|f+1\|_{p^{\prime}(x, t)} \cdot\|\xi\|_{p(x, t)} \\
\leq & 2 \max \left\{F_{1}, F_{2}\right\}\|\nabla \xi\|_{p(x, t)}+2 \max \left\{F_{3}, F_{4}\right\}\|\xi\|_{p(x, t)} \\
\leq & \left(2\left(\left(K^{2}(T)+1\right)^{\sigma / 2}+d_{0}\right)^{\frac{1}{p^{ \pm}-1}} K(T)|\Omega|+2|f+1|_{\infty}|T|\right)\|\xi\|_{W\left(Q_{T}\right)},
\end{aligned}
$$

where

$$
\begin{aligned}
& F_{1}=\left(\int_{0}^{T} \int_{\Omega}\left\{\left[\left(u_{\varepsilon}^{\sigma}+\varepsilon^{2}\right)^{\sigma / 2}+d_{0}\right]\left|\nabla u_{\varepsilon}\right|^{p(x, t)-1}\right\}^{\frac{p(x, t)}{p(x, t)-1}} \mathrm{~d} x \mathrm{~d} t\right)^{\frac{1}{p^{\prime}+}}, \\
& F_{2}=\left(\int_{0}^{T} \int_{\Omega}\left\{\left[\left(u_{\varepsilon}^{\sigma}+\varepsilon^{2}\right)^{\sigma / 2}+d_{0}\right]\left|\nabla u_{\varepsilon}\right|^{p(x, t)-1}\right\}^{\frac{p(x, t)}{p(x, t)-1}} \mathrm{~d} x \mathrm{~d} t\right)^{\frac{1}{p^{\prime}-}}, \\
& F_{3}=\left(\int_{0}^{T} \int_{\Omega}|f|^{p^{\prime}(x, t)} \mathrm{d} x \mathrm{~d} t\right)^{\frac{1}{p^{\prime}+}}, \quad F_{4}=\left(\int_{0}^{T} \int_{\Omega}|f+1|^{p^{\prime}(x, t)} \mathrm{d} x \mathrm{~d} t\right)^{\frac{1}{p^{\prime}-}} .
\end{aligned}
$$

Then (38) follows from Lemma 3.5.

From [6] we can get the following inclusions:

$$
\begin{aligned}
& u_{\varepsilon} \in W\left(Q_{T}\right) \subseteq L^{p^{-}}\left(0, T ; W_{0}^{1, p^{-}}(\Omega)\right), \quad u_{\varepsilon t} \in W^{\prime}\left(Q_{T}\right) \subseteq L^{\frac{p^{+}}{p^{+}-1}}\left(0, T ; V_{+}(\Omega)\right), \\
& W_{0}^{1, p^{-}}(\Omega) \subset L^{2}(\Omega) \subset V_{+}^{\prime}(\Omega) \quad \text { with } V_{+}(\Omega)=\left\{u(x)\left|u \in L^{2}(\Omega) \cap W_{0}^{1,1}(\Omega),\right| \nabla u \mid \in L^{p^{+}}\right\} .
\end{aligned}
$$

These conclusions, together with the uniform estimates in $\varepsilon$, allow us to extract from the sequence $\left\{u_{\varepsilon}\right\}$ a subsequence (for simplicity, we assume that it merely coincides with the whole sequence) such that

$$
\begin{cases}u_{\varepsilon} \rightarrow u & \text { a.e. in } Q_{T}, \\ \nabla u_{\varepsilon} \rightarrow \nabla u & \text { weakly in } L^{p(x, t)}\left(Q_{T}\right), \\ u_{\varepsilon}^{\sigma}\left|\nabla u_{\varepsilon}\right|^{p(x, t)-2} D_{i} u_{\varepsilon} \rightarrow A_{i}(x, t) & \text { weakly in } L^{p^{\prime}(x, t)}\left(Q_{T}\right), \\ \left|\nabla u_{\varepsilon}\right|^{p(x, t)-2} D_{i} u_{\varepsilon} \rightarrow W_{i}(x, t) & \text { weakly in } L^{p^{\prime}(x, t)}\left(Q_{T}\right)\end{cases}
$$

for some functions $u \in W\left(Q_{T}\right), A_{i}(x, t) \in L^{p^{\prime}(x, t)}\left(Q_{T}\right)$, and $W_{i}(x, t) \in L^{p^{\prime}(x, t)}\left(Q_{T}\right)$.

Lemma 3.7 For almost all $(x, t) \in Q_{T}$,

$$
\lim _{\varepsilon \rightarrow 0^{+}} \iint_{Q_{T}}\left(\left(u_{\varepsilon}^{2}+\varepsilon^{2}\right)^{\frac{\sigma}{2}}-u_{\varepsilon}^{\sigma}\right)\left|\nabla u_{\varepsilon}\right|^{p(x, t)-2} \nabla u_{\varepsilon} \nabla \xi \mathrm{d} x \mathrm{~d} t=0, \quad \forall \xi=W\left(Q_{T}\right) .
$$


Proof We first compute

$$
\begin{aligned}
I & \triangleq \iint_{Q_{T}}\left(\left(u_{\varepsilon}^{2}+\varepsilon^{2}\right)^{\frac{\sigma}{2}}-u_{\varepsilon}^{\sigma}\right)\left|\nabla u_{\varepsilon}\right|^{p(x, t)-2} \nabla u_{\varepsilon} \nabla \xi \mathrm{d} x \mathrm{~d} t \\
& =\frac{\sigma}{2} \varepsilon^{2} \iint_{Q_{T}}\left(\int_{0}^{1}\left(u_{\varepsilon}^{2}+s \varepsilon^{2}\right)^{\frac{\sigma-2}{2}} d s\right)\left|\nabla u_{\varepsilon}\right|^{p(x, t)-2} \nabla u_{\varepsilon} \nabla \xi \mathrm{d} x \mathrm{~d} t \\
& \leq \sigma \varepsilon^{2}\left(K^{2}(T)+1\right)^{\frac{\sigma-2}{2}}\left\|\left|\nabla u_{\varepsilon}\right|^{p(x, t)-1}\right\|_{p^{\prime}(x, t)}\|\nabla \xi\|_{p(x, t)} \\
& \leq C \varepsilon^{2} \max \left\{\left(\iint_{Q_{T}}\left|\nabla u_{\varepsilon}\right|^{p(x, t)} \mathrm{d} x \mathrm{~d} t\right)^{\frac{p^{+}-1}{p^{+}}},\left(\iint_{Q_{T}}\left|\nabla u_{\varepsilon}\right|^{p(x, t)} \mathrm{d} x \mathrm{~d} t\right)^{\frac{p^{-}-1}{p^{-}}}\right\}\|\nabla \xi\|_{p(x, t)} .
\end{aligned}
$$

By (35) we get

$$
I \leq C \varepsilon^{2-\frac{\sigma\left(p^{+}-1\right)}{p^{+}}}\|\nabla \xi\|_{p(x, t)}
$$

Passing to the limit as $\varepsilon \rightarrow 0$, we obtain Lemma 3.7.

Lemma 3.8 For almost all $(x, t) \in Q_{T}$, we have

$$
A_{i}(x, t)=u^{\sigma} W_{i}(x, t), \quad i=1,2, \ldots, N
$$

Proof In (39), letting $\varepsilon \rightarrow 0$, we have

$$
\begin{gathered}
\iint_{Q_{T}} u_{\varepsilon}^{\sigma}\left|\nabla u_{\varepsilon}\right|^{p(x, t)-2} \nabla u_{\varepsilon} \nabla \xi \mathrm{d} x \mathrm{~d} t \rightarrow \sum \iint_{Q_{T}} A_{i}(x, t) D_{i} \xi \mathrm{d} x \mathrm{~d} t \\
\iint_{Q_{T}} u_{\varepsilon}^{\sigma}\left|\nabla u_{\varepsilon}\right|^{p(x, t)-2} \nabla u_{\varepsilon} \nabla \xi \mathrm{d} x \mathrm{~d} t \rightarrow \sum \iint_{Q_{T}} W_{i}(x, t) D_{i} \xi \mathrm{d} x \mathrm{~d} t .
\end{gathered}
$$

By Lebesgue's dominated convergence theorem we have

$$
\lim _{\varepsilon \rightarrow 0} \sum_{i=1}^{N} \iint_{Q_{T}}\left(u_{\varepsilon}^{\sigma}-u^{\sigma}\right) A_{i}(x, t) D_{i} \xi \mathrm{d} x \mathrm{~d} t=0 .
$$

So

$$
\begin{aligned}
& \lim _{\varepsilon \rightarrow 0} \sum \iint_{Q_{T}}\left(u_{\varepsilon}^{\sigma}\left|\nabla u_{\varepsilon}\right|^{p(x, t)-2} D_{i} u_{\varepsilon}-u^{\sigma} W_{i}(x, t)\right) D_{i} \xi \mathrm{d} x \mathrm{~d} t \\
& \quad=\lim _{\varepsilon \rightarrow 0} \iint_{Q_{T}}\left(u_{\varepsilon}^{\sigma}-u^{\sigma}\right)\left|\nabla u_{\varepsilon}\right|^{p(x, t)-2} D_{i} u_{\varepsilon}+u^{\sigma}\left(\left|\nabla u_{\varepsilon}\right|^{p(x, t)-2} D_{i} u_{\varepsilon}-W_{i}(x, t)\right) D_{i} \xi \mathrm{d} x \mathrm{~d} t \\
& \quad=0 .
\end{aligned}
$$

By (40)-(42) and the previous inequalities, we complete the proof of Lemma 3.8.

Lemma 3.9 For almost all $(x, t) \in Q_{T}$, we have

$$
W_{i}(x, t)=\left|\nabla u_{\varepsilon}\right|^{p(x, t)-2} D_{i} u, \quad i=1,2, \ldots, N
$$


Proof In (8), choosing $\xi=\left(u_{\varepsilon}-u\right) \Phi$ with $\Phi \in W\left(Q_{T}\right), \Phi \geq 0$, we have

$$
\begin{aligned}
& \iint_{Q_{T}}\left[u_{\varepsilon t}\left(u_{\varepsilon}-u\right) \Phi+\Phi\left(u_{\varepsilon}^{\sigma}+d_{0}\right)\left|\nabla u_{\varepsilon}\right|^{p(x, t)-2} \nabla u_{\varepsilon} \nabla\left(u_{\varepsilon}-u\right)\right] \mathrm{d} x \mathrm{~d} t \\
& +\iint_{Q_{T}}\left[\left(u_{\varepsilon}-u\right)\left(u_{\varepsilon}^{\sigma}+d_{0}\right)\left|\nabla u_{\varepsilon}\right|^{p(x, t)-2} \nabla u_{\varepsilon} \nabla \Phi-f(x, t)\left(u_{\varepsilon}-u\right) \Phi\right] \mathrm{d} x \mathrm{~d} t \\
& +\iint_{Q_{T}}\left(\left(u_{\varepsilon}^{\sigma}-\varepsilon^{2}\right)^{\frac{\sigma}{2}}-u_{\varepsilon}^{\sigma}\right)\left|\nabla u_{\varepsilon}\right|^{p(x, t)-2} \nabla u_{\varepsilon} \nabla \xi \mathrm{d} x \mathrm{~d} t=0 .
\end{aligned}
$$

It follows that

$$
\lim _{\varepsilon \rightarrow 0} \iint_{Q_{T}} \Phi\left(u_{\varepsilon}^{\sigma}+d_{0}\right)\left|\nabla u_{\varepsilon}\right|^{p(x, t)-2} \nabla u_{\varepsilon} \nabla\left(u_{\varepsilon}-u\right) \mathrm{d} x \mathrm{~d} t=0 .
$$

On the other hand, from $u_{\varepsilon}, u \in L^{\infty}\left(Q_{T}\right)$ and $|\nabla u| \in L^{p(x, t)}\left(Q_{T}\right)$ we get

$$
\begin{aligned}
& \lim _{\varepsilon \rightarrow 0} \iint_{Q_{T}} \Phi\left(u^{\sigma}+d_{0}\right)|\nabla u|^{p(x, t)-2} \nabla u \nabla\left(u_{\varepsilon}-u\right) \mathrm{d} x \mathrm{~d} t=0, \\
& \lim _{\varepsilon \rightarrow 0} \iint_{Q_{T}} \Phi\left(u_{\varepsilon}^{\sigma}+u^{\sigma}\right)\left|\nabla u_{\varepsilon}\right|^{p(x, t)-2} \nabla u_{\varepsilon} \nabla\left(u_{\varepsilon}-u\right) \mathrm{d} x \mathrm{~d} t=0 .
\end{aligned}
$$

Note that

$$
\begin{aligned}
0 \leq & \left(\left|\nabla u_{\varepsilon}\right|^{p(x, t)-2} \nabla u_{\varepsilon}-|\nabla u|^{p(x, t)-2} \nabla u\right) \nabla\left(u_{\varepsilon}-u\right) \\
\leq & \frac{1}{d_{0}}\left[\left(u_{\varepsilon}^{\sigma}+d_{0}\right)\left|\nabla u_{\varepsilon}\right|^{p(x, t)-2} \nabla u_{\varepsilon}-\left(u_{\varepsilon}^{\sigma}-u^{\sigma}\right)\left|\nabla u_{\varepsilon}\right|^{p(x, t)-2} \nabla u\right] \nabla\left(u_{\varepsilon}-u\right) \\
& -\frac{1}{d_{0}}\left(u_{\varepsilon}^{\sigma}+d_{0}\right)\left|\nabla u_{\varepsilon}\right|^{p(x, t)-2} \nabla u \nabla\left(u_{\varepsilon}-u\right) .
\end{aligned}
$$

By (44)-(46) we have

$$
\lim _{\varepsilon \rightarrow 0} \iint_{Q_{T}} \Phi\left(\left|\nabla u_{\varepsilon}\right|^{p(x, t)-2} \nabla u_{\varepsilon}-\left|\nabla u_{\varepsilon}\right|^{p(x, t)-2} \nabla u\right) \nabla\left(u_{\varepsilon}-u\right) \mathrm{d} x \mathrm{~d} t=0
$$

Lemma 3.10 As $\varepsilon \rightarrow 0$,we have

$$
\beta_{\varepsilon}\left(u_{\varepsilon}-u_{0}\right) \rightarrow \xi \in G\left(u-u_{0}\right)
$$

Proof Using (7) and the definition of $\beta_{\varepsilon}$, we have

$$
\beta_{\varepsilon}\left(u_{\varepsilon}-u_{0}\right) \rightarrow \xi \quad \text { as } \varepsilon \rightarrow 0 .
$$

Now we prove that $\xi \in G\left(u-u_{0}\right)$. According to the definition of $G(\cdot)$, we only need to prove that if $u\left(x_{0}, t_{0}\right)>u_{0}\left(x_{0}\right)$, then $\xi\left(x_{0}, t_{0}\right)=0$. In fact, if $u\left(x_{0}, t_{0}\right)>u_{0}(x)$, there exist a constant $\lambda>0$ and a $\delta$ neighborhood $B_{\delta}\left(x_{0}, t_{0}\right)$ such that if $\varepsilon$ is small enough, we have

$$
u_{\varepsilon}(x, t) \geq u_{0}(x)+\lambda, \quad \forall(x, t) \in B_{\delta}\left(x_{0}, t_{0}\right) .
$$


Thus, if $\varepsilon$ is small enough, then we have

$$
0 \geq \beta_{\varepsilon}\left(u_{\varepsilon}-u_{0}\right) \geq \beta_{\varepsilon}(\lambda)=0, \quad \forall(x, t) \in B_{\delta}\left(x_{0}, t_{0}\right) .
$$

Furthermore, it follows by $\varepsilon \rightarrow 0$ that

$$
\xi(x, t)=0, \quad \forall(x, t) \in B_{\delta}\left(x_{0}, t_{0}\right) .
$$

Hence, (48) holds, and the proof of Lemma 3.10 is completed.

$$
\text { Applying (28), (29), and Lemma 3.10, it is clear that }
$$

$$
u(x, t) \leq u_{0}(x) \quad \text { in } \Omega_{T}, \quad u(x, 0)=u_{0}(x) \quad \text { in } \Omega, \xi \in G\left(u-u_{0}\right),
$$

and thus (a), (b), and (c) hold. The remaining arguments of the existence part are the same as those of Theorem 2.1 in [8], and we omit the details. Moreover, the uniqueness of solutions can be proved by repeating Lemma 3.1.

\section{Acknowledgements}

This work was supported by Scientific research Foundation of Shaanxi Railway Institute (No. KY2016-04) and Natural Science Foundation of Shaanxi Provincial Department of Education (No. 17JK0170). The author is sincerely grateful to the reference and the Associate Editor handling the paper for their valuable comments.

\section{Competing interests}

The author declares that there is no conflict of interests regarding the publication of this paper.

\section{Authors' contributions}

The author read and approved the final manuscript.

\section{Publisher's Note}

Springer Nature remains neutral with regard to jurisdictional claims in published maps and institutional affiliations.

Received: 7 August 2017 Accepted: 29 January 2018 Published online: 08 February 2018

\section{References}

1. Chen, X., Yi, F., Wang, L.: American lookback option with fixed strike price 2-D parabolic variational inequality. J. Differ. Equ. 251, 3063-3089 (2011)

2. Zhou, Y., Yi, F.: A free boundary problem arising from pricing convertible bond. Appl. Anal. 89, 307-323 (2010)

3. Chen, X., Yi, F.: Parabolic variational inequality with parameter and gradient constraints. J. Math. Anal. Appl. 385, 928-946 (2012)

4. Zhou, Y., Yi, F.: A variational inequality arising from American installment call options pricing. J. Math. Anal. Appl. 357, 54-68 (2009)

5. Sun, Y., Shi, Y., Gu, X.: An integro-differential parabolic variational inequality arising from the valuation of double barrier American option. J. Syst. Sci. Complex. 27, 276-288 (2014)

6. Sun, Y., Shi, Y., Wu, M.: Second-order integro-differential parabolic variational inequalities arising from the valuation of American option. J. Inequal. Appl. 2014, 8 (2014)

7. Sun, Y., Shi, Y.: The existence of solution to a class of degenerate parabolic variational inequality. J. Inequal. Appl. 2015, 204 (2015)

8. Gao, B., Gao, W.: Study of weak solutions for parabolic equations with nonstandard growth conditions. J. Math. Anal. Appl. 374, 374-384 (2011) 